\title{
SOME MATHEMATICAL ASPECTS OF THE NEW MECHANICS*
}

BY TULLIO LEVI-CIVITA

1. The Antecedent of Any Mechanical Conception. The Moving Point. The mental pictures of natural phenomena are of very different kinds. Among the simplest and most basic of these mental pictures we may include the kinematical model of the motion of a geometrical point. Such a picture is an abstraction, i.e., an ideal construction, which may be obtained conveniently through axiomatic treatment of geometry and time.

The use of this model for real bodies necessitates, of course, a further physical postulate, or axiom, quite independent of previous kinematical assumptions. But it is so intuitively evident, in common-life scale, that no thinker has till now felt the need of elucidating or criticizing its adoption. We may designate it as the "axiom of substantiality of matter," or more briefly, of Descartes, if we agree to give this precise meaning to the famous assertion of Descartes "tout se fait par figure et par mouvement." Indeed these words have been rather interpreted as generally alluding to a mechanical explanation of nature. Such an explanation, for a great body of facts (including terrestrial and celestial motion), was offered by Newton and his successors, and prevailed undisputed in the 18 th century.

2. Autonomous Phenomenological Theories. In the following century new classes of phenomena were detected and thoroughly investigated, especially the propagation of heat and electromagnetism, for which autonomous theories were established which were entirely satisfactory as representations of experience and highly valuable from a mathematical point of view, but which were entirely or almost independent of mechanical framework. I need hardly mention the classical treatises of Fourier and Maxwell. Such phenomenological, even somewhat detached,

* An address delivered, by invitation of the authorities of the Century of Progress Exposition and the American Association for the Advancement of Science, in Chicago, June 20,1933, before a joint meeting of the American Mathematical Society and Section A of the A.A.A.S. 
theories seemed at the end of the last century (and may perhaps still seem) excellent as far as they offer a quantitatively exact description of observed circumstances, and are otherwise capable of being used for systematic predictions.

3. Relativity and Efforts toward Unified Formulation of all Physics. A little later Einstein's relativity showed, in a wonderful though unexpected way, an intimate linkage between laws of motion, gravitation, and geometrical optics. Weyl, Eddington, Einstein himself, and many, many others were led to search for a more general unified view which would include electromagnetism and therefore physical optics.

4. Quantum Mechanics. Early Stage (Bohr-Sommerfeld). Meanwhile atomic physics began, this name belonging to the imposing congeries of investigations - permit me to name only Rutherford and Millikan - on radiation, rays, and isolated particles which verified the existence of minute constituents of electricity and matter. Up to a certain point the usual principles were still applicable, permitting rough estimates, but the systematic use of Planck's quantum of action $h$ demanded a modified attitude towards the classical laws of motion. The differential equations expressing them were not changed, but the constants of integration, or rather some of them (always including energy), were deprived of continuity, and supposed to assume only discrete values (integral multiples of the lowest). By regarding the atom of hydrogen as made up of a single negative electron revolving round a positive nucleus, Bohr succeeded in presenting to the astonished world a mechanical model of spectroscopic lines (Balmer's, Lyman's, and Paschen's series) of very high accuracy.

More generally the theory of adiabatic invariants (Ehrenfest, Epstein, Burgers), systematically developed by Sommerfeld, led to an exact or approximate model of other atoms (in concept we may say of all other atoms), with the same degree of accuracy.

This was hardly accounted for in the classical work of Sommerfeld, Atombau und Spektrallinien, when a more severe criticism of old facts and some new discoveries led to new foundations of quantum mechanics.

5. The Failure of the Basic Postulate of Kinematical Substantiality. We shall speak of the new mechanics in a few minutes; 
but I wish now to call your attention to a singular event, the most curious, in my opinion, that has ever been recorded in the evolution of scientific points of view. As you know, it was only in the first years of the present century that the real existence of small particles like electrons and atoms was experimentally ascertained. Formerly the great majority of scientists were doubtful, or at least reserved in opinion, concerning the question "to be or not to be" of atoms, which was then metaphysical in the literal meaning of the word, i.e., beyond the means of control available at that time. But no one had suspected that such things, if they existed, did not obey the cartesian postulate of substantiality, nor that for the description of the main phenomena connected with them we would be compelled to give up the most ancient and familiar device for the study of the physical world: the merely kinematical concept of a moving geometrical point.

In fact new experiments have on the one hand afforded irrefutable evidence for the existence of something-atom, electron, or photon-having individual characteristics, at a certain place (representable by a geometrical point), at a given instant $t$. But on the other hand it was ascertained that these entities have so little consistency that they are essentially modified, sometimes even (in a certain sense) annihilated, by our observations. If such an observation has taken place at a given instant $t$, a further observation is influenced: the latter does not give a sufficient account of, say, the electron, inasmuch as the electron has been strongly affected by the former observation.

6. Heisenberg's Principle of Indeterminacy. Analogous difficulties occur, of course, if we try to make observations at two very near instants, or, as a limiting process, to measure at the same instant position and speed. Furthermore a careful discussion of the (mainly) optical devices furnishing these evaluations brought Heisenberg to the discovery of his concrete principle of indeterminacy (or uncertainty), which approximates the second law of thermodynamics in affirming some impossibility in our physical world: the spontaneous passage of heat to a hotter body, in thermodynamics, and, in atomic physics, the measurement of both position and velocity with absolute accuracy. Obviously the absolute accuracy alluded to has usually 
only the potential meaning of an accuracy which might theoretically be increased at will. More precisely this indeterminacy principle states, for one degree of freedom, that if $x$ designates the space coordinate of an electron (or photon), $m$ its mass, $v$ its velocity, and $p=m v$ its momentum, the inaccuracies $\Delta x, \Delta p$ are unavoidably of such amounts as to render their product

$$
\Delta x \cdot \Delta p>h,
$$

where $h=(6.5) 10^{-27}$ in C.G.S. units is the Planck's constant (elementary quantum of action).

For ordinary particles of matter in bulk, even very minute, say $m$ as small as $10^{-3} \mathrm{gr}$. (a milligram-mass), the inequality (1), since $p=m v$, gives for the kinematical indetermination

$$
\Delta x \cdot \Delta v>h / m>(6.5) 10^{-24} \mathrm{~cm} .{ }^{2} / \mathrm{sec} .
$$

This is quite compatible with the greatest exactness available or desirable in macromechanics. But the matter is quite different for much smaller particles like electrons. The order of magnitude of $m$ is then $10^{-27} \mathrm{gr}$., therefore $h / m$ becomes a value of the order of 6.5 , and the product of kinematical uncertainties $\Delta x \cdot \Delta v$ has to be expected to be considerably greater than unity:

$$
\Delta x \cdot \Delta v>1,
$$

which deprives the corresponding physical observations of any interest. In fact, the linear dimensions of an electron being of the order of $10^{-13}$, a not illusory $\Delta x$ must be less than this, and then $\left(1^{\prime}\right)$ requires

$$
\Delta v>10^{13} \mathrm{~cm} . / \mathrm{sec} .
$$

which is more than a hundred times the velocity of light, $3\left(10^{10}\right)$. Because of such an indeterminacy no useful information may be obtained about velocity.

In conclusion, atoms, electrons, photons do exist, since several phenomena grant physical evidence of them, but the representation of them as moving particles (or even as a system of moving bodies) is not adequate, because the indications which real experimentation may supply along this line are too vague.

7. Digression on Rays as Filiform Flow of Electricity. If, however, the elementary kinematical (and in consequence any dy- 
namical) description must be abandoned as far as a single electron is concerned, Heisenberg's principle does not entirely exclude another model capable of furnishing statistical information on real observable average effects.

For this purpose we must imagine not a single electron but a great many, apparently forming a current, e.g., a ray. To explore the field of velocity interior to the ray (which may be some centimeters in length), we may take, or think of taking, an experimental measure of velocity, say, in every cubic micron of the space occupied by the ray. Since $\Delta x$ is here $10^{-4} \mathrm{~cm}$., formula $\left(1^{\prime}\right)$ gives for the measured velocity an inaccuracy certainly greater than $10^{4} \mathrm{~cm}$./ $/ \mathrm{sec}$., but with a possibility of becoming less than, say, $10^{5}$, i.e., $1 \mathrm{~km}$. $/ \mathrm{sec}$. This is a very satisfactory approximation for velocities comparable to that of light (i.e., for velocity over one tenth that of light). It follows from this estimate that the treatment of electric radiations ( $\beta$-rays and the like) as a continuous flow is quite compatible with the principle of indeterminacy, and therefore even today may be used satisfactorily as a theoretical scheme to investigate such average properties of rays (e.g., form deviations, forces exerted on and by them) for which a continuous or discrete distribution of charges (electrons) are practically indistinguishable.

The logical scheme is the following: no physical reality may be attached (on an atomic scale) to the motion of a single electron; on the other hand a field theory of the continuous flow of such particles (essentially a local description in the most elementary Eulerian form) may be fairly well supplied by concrete experiences. Now, still relying on these experimental measures, there is nothing to prevent us from substituting in our mathematical representation for the Eulerian point of view that of Lagrange, that is, to resolve the flow into (hypothetic) elementary moving particles, or even to replace their continuous flow by a discrete bombardment of very minute projectiles, occurring frequently enough to produce (on the above approximation) the same mean effects. And we may even suppose that such projectiles have just the mass of a single electron. In this way it becomes possible, and very interesting, to give a positive meaning to the motion of a great many electrons or photons forming rays of different kinds.

In this connection I may perhaps be allowed to add a per- 
sonal remark. As you certainly remember, the realization that $\beta$-rays were composed of electrons (charges of negative electricity without material support) was reached in 1902 by Kaufmann's experiments, which were in surprising agreement with a suggestive ballistic theory of Abraham (whose rigid electron was a mathematical and mechanical refinement of a simpler relativistic model of Lorentz). This conclusion, as far as the purely electrical constitution is concerned, was afterwards verified by other facts. But, as we have seen, by Heisenberg's principle, no ballistic scheme has, in this range, direct physical meaning. However, in 1909 I proposed an asymptotic theory of continuous flow which leads to the same physically controlled consequences (and to one more not yet tested) and which, unlike the former, is consistent even today.*

8. Validity of Classical Physics for Estimates of Mean Values. Sone other facts concerning mean values are satisfactorily explained by this very elementary process of averaging through continuous flow. The Compton effect belongs to this class; and we may in this way understand the success of the very elementary corpuscular explanation (based on conservation of both momentum and energy) offered by Compton himself and Debye. Actually, as previously observed, the uncertainty principle prevents us from treating photons or electrons as isolated particles; but the diffusion of X-rays in a gas by electrons, which gives rise to the Compton effect, is just recognizable as a continuous flow. Then a further treatment of this flow as discontinuous and energetically quantized is justified as indicated in $\$ 7$, and brings us again to the elementary explanation just mentioned. From this the very instructive assumption may be made that, as far as observable means are concerned, principles and therefore formulas of classical mechanics are still available, at least to within a certain degree of precision. For a further refinement, and a nearer approach to phenomena on an individual scale, other devices must be sought.

9. The New Principles of Quantum Mechanics and Wave Mechanics Inferred from a Typical Case (Hydrogen Atom). A direct analysis of experimental data, especially in spectroscopy, where

* Teoria Asintotica delle Radiazioni Elettriche, Rendiconti della Accademia dei Lincei, vol. 18 (1st sem. 1909), pp. 83-93. 
only lines (frequencies) and intensities of these lines are observable, was claimed by Born, Jordan, and Heisenberg. They succeeded in building a satisfactory theory involving only an enumerable set of directly measurable parameters, and based, from a mathematical point of view, on the algebra of matrices.

A quite different theory, equivalent as regards positive results, but much closer, at least in the analytical scaffolding, to the old captivating models of mathematical physics, was soon after proposed and elaborated by Schrödinger.

He first took into consideration the hydrogen atom, searching for a partial differential equation of common type (hence linear, self-adjoint of the second order) admitting levels of energy (quantized values of energy) as characteristic values.

Schrödinger's guiding principle has been to express just in this form de Broglie's association of waves with ordinary corpuscular dynamics, the wave function $\psi$ being strictly correlated with that principal function $V$ of Hamilton, which would belong to the electron of a hydrogen atom, if corpuscular nonrelativistic treatment were allowed.

The result is remarkably simple. Let

$$
H(p \mid x)=E
$$

be the equation of the first order defining $V$, where $p$ generally replaces the set

$$
p_{1}=\partial V / \partial x^{1}, p_{2}=\partial V / \partial x^{2}, \cdots, p_{n}=\partial V / \partial x^{n},
$$

and $x$ the set

$$
x^{1}, x^{2}, \cdots, x^{n}
$$

of the independent variables; in the present case $n$ has to be put $=3$.

Schrödinger's operator $S$ to be applied to $\psi$ is simply $H-E$ itself, provided that in $H$ every $p_{k}$ has the operational meaning $(h /(2 \pi i)) \partial / \partial x^{k}$. The linear equation in $V$ being thus

$$
S \psi \equiv[H\{(h /(2 \pi i)) \partial / \partial x \mid x\}-E] \psi=0,
$$

the correct energy levels appeared as characteristic values of (3), and moreover, assuming

$$
\psi=e^{2 \pi i V / h},
$$

the Hamilton-Jacobi equation (3) follows for $V$, up to the first order of approximation with respect to $h$. 
10. First Attempt at a Mathematical Correlation between Waves and Particles. Born's Appeal to the Calculus of Probability. The last conclusion was for a while regarded as capable of furnishing a well defined association between the motion of a particle in the field considered and a wave-packet surrounding the particle and perceptible only in the immediate neighborhood of the paticle itself. But a further study has shown that difficulties arise on presenting in detail any such correlation; above all it does not harmonize with Heisenberg's indeterminacy.

To suppress fundamental discrepancies of this sort, Born made a suggestion of wide philosophical application. Instead of connecting the wave-function $\psi$ with some corpuscular entity, belonging to or associated with actually observed facts, he proposed an interpretation borrowed from statistical mechanics. More exactly, he substituted for Schrödinger's first assumption that $|\psi|^{2}$ should be proportional to a real electrical density, a less binding and highly fruitful probability interpretation, namely, that $|\psi|^{2} d S$ is available as a distribution function, in the sense that $|\psi|^{2} d S(d S$ element of space) must be interpreted as the probability for the electron [or rather for an electron whose energetic and spectroscopic apparencies are correctly described by (2) or (3) ] to be present in $d S$ at the moment considered. This implies of course that $\int_{S}|\psi|{ }^{2} d S$ (the probability that the electron finds itself somewhere in the whole space) is a certainty, that is $=1$, a condition which is easily fulfilled by means of the constant factor which, on account of the linearity of (3), certainly remains at our disposal.

As in the kinetic theory, once in possession of the law of distribution, we are able to obtain probable values of any physical quantity, connected statistically with a very large number of others having the same macroscopic behavior.

11. Relativistic Wave-Equation (for a Single Particle Moving in a Given Field). According to Einstein's geodetic principle, the motion of a particle whose mass is $m$ and electric charge $e$, under gravitational and electromagnetic forces of any kind, may be represented by a canonical system. Its Hamiltonian is*

\footnotetext{
* For the deduction of this Hamiltonian from the Einstein-De Donder variational postulate see Appendix I.
} 


$$
H=(1 / 2) \sum_{0}^{3}{ }_{\mu \nu} g^{\mu \nu}\left(p_{\mu}-e \phi_{\mu} / c\right)\left(p_{\nu}-e \phi_{\nu} / c\right)-m^{2} c^{2} / 2
$$

where $g^{\mu \nu}$ denotes the (contravariant) metric tensor, $\phi_{\mu}$ the covariant electromagnetic potential, referred, like $e$, to electrostatic units; $c$ the light velocity in vacuo, and $p_{\mu}$ the momentum, connected with the space-time coordinate $x^{\mu}$. As usual, we shall regard $x^{0}$ as the time variable, $x^{1}, x^{2}, x^{3}$ as space variables. In the absence of a gravitational field, the coordinates may be supposed pseudo-cartesian, and then the $g^{\mu \nu}$ have, like $g_{\mu \nu}$, their pseudo-euclidean values:

$$
\begin{array}{rrrr}
1 & 0 & 0 & 0 \\
0 & -1 & 0 & 0 \\
0 & 0 & -1 & 0 \\
0 & 0 & 0 & -1
\end{array}
$$

The canonical system, having (5) as Hamiltonian function, admits in any case the integral $H=$ const.; we must take more strictly

$$
H(p \mid x)=0,
$$

as a consequence of the transformation which gives canonical form to the original principle of variation.

Schrödinger's relativistic equation may be written at once, extending the formal rule of $\$ 9$ : substitution of the operator $(h /(2 \pi i)) \partial / \partial x^{\mu}$ for $p_{\mu}$. The only generalization consists in replacing the ordinary $\partial / \partial x^{\mu}$ by the covariant derivation $\nabla_{\mu}$ (with reference to $\left.d s^{2}=g_{\mu \nu} d x^{\mu} d x^{\nu}\right)$. We have, therefore, putting for brevity

$$
h^{\prime}=h /(2 \pi),
$$

omitting, as does Einstein, the symbol for summation, and multiplying the two sides by $-2 h^{\prime 2}$, Schrödinger's relativistic equation

$$
\begin{aligned}
S \psi & \equiv\left[g^{\mu \nu}\left(\nabla_{\mu}-i e \phi_{\mu} /\left(c h^{\prime}\right)\right)\left(\nabla_{\nu}-i e \phi_{\nu} /\left(c h^{\prime}\right)\right)+m^{2} c^{2} / h^{\prime 2}\right] \psi \\
& =0
\end{aligned}
$$

sometimes known as Gordon's equation.

In the absence of any (gravitational and electromagnetic) field, referring to pseudo-cartesian coordinates and putting 
$\psi=e^{i(m c+E / c) x^{0} / h^{\prime}} \Psi$, where $\Psi$ is independent of $x^{0}$ and $E$ is a constant energy, we have simply

$$
-\sum_{1}^{3} \partial^{2} \Psi /\left(\partial x^{\mu}\right)^{2}+\left\{m^{2} c^{2}-(m c+E / c)^{2}\right\} \Psi / h^{\prime 2}=0
$$

or even, if $E$ is very small in comparison with $m c^{2}$,

$$
\sum_{1}^{3} \partial^{2} \Psi /\left(\partial x^{\mu}\right)^{2}+2 m E \Psi / h^{\prime 2}=0,
$$

the non-relativistic equation of Schrödinger.

12. Spin. Pauli-Dirac Wave-Vector. For a short time it was believed that (in the foreseen range) equation (8), which had been used with imposing success, should be definitive. But, while fine structure was satisfactorily included, striking observations were soon made concerning spin effect and magnetic disturbances (anomalous Zeeman-effect), which did not agree with the consequences of (8) as far as spectroscopic displacements are concerned.

A very interesting synthetic reasoning along the line of group theory brought Pauli to introduce the needed correction as Goudsmith and Ullenbeck had already done for Sommerfeld's corpuscular theory. Pauli substituted for (8) a system of two equations involving two wave-functions. A further improvement was made by Dirac through a thorough analysis and original formal translation of physical assumptions. This author has succeeded, we may say, in splitting up Pauli's differential equation of the second order into four equations of the first order with as many unknown functions $\psi_{0}, \psi_{1} ; \psi_{2}, \psi_{3}$. This quadruple may be interpreted as a vector $\psi$ in space-time. Dirac and his followers did not make this interpretation. They gave to the set of wave-functions $\psi_{\mu}(\mu=0,1,2,3)$ another interpretation deduced from the theory of representation of the Lorentz group and the so-called spinors.

In terms of Dirac's $\psi$ 's the real quantity

$$
\sum_{0}^{3} \psi_{s} \psi_{s}^{*}
$$

(star denoting passage to the conjugate imaginary) is to be interpreted as (probable) electric density in ordinary three-dimensional space. 
Dirac's equations proved to be in agreement with the more refined experiences just mentioned, reproducing, if spin-correction is neglected, Schrödinger's equation as resolvent of the second order.

Dirac's line of thought, in both geometric and algebraic points of view, is strictly related to ordinary (pseudo-euclidean) space-time and to pseudo-cartesian coordinates, leaving, therefore, gravitation unaccounted for.

13. General Coordinates. Gravitational Field. Criticism of Matrix Equations. General methods of absolute differential calculus allow, of course, translation to general coordinates of any system given in particular variables. Many authors applied themselves to this task; a more recent attempt was made by Schrödinger, $\dagger$ De Donder and Miss Dupont, $\ddagger$ and myself. $\S$ Following Schrödinger I have worked out the most natural (in a certain sense even uniquely determined) extension not only to general coordinates, but even to any relativistic $d s^{2}$, including gravitational actions.

To reach this extension I have been forced (like Schrödinger and many others) to introduce a lattice, i.e., a system of four mutually perpendicular congruences of lines, in space-time. This lattice is not an intrinsic feature of the physical phenomenon; it has only an auxiliary role, and must therefore disappear from final equations, if properly treated. An illustration of a similar circumstance in well known more elementary instances is shown in Appendix II.

It appeared from my calculations that in the generalized Dirac's equations a residual influence of the auxiliary lattice is not to be avoided, and this is quite unreasonable, the lattice having been introduced as a mathematical tool without any reference to the atomic events.

Indeed Dirac's original equations for relativistic electrons in pseudo-euclidean space (i.e. in absence of gravitation) involve a set of 4-rowed numerical matrices. Their geometric counter-

$\dagger$ Diracsches Elecktron im Schwerefeld, Berichte der Preussischen Akademie, 1932, pp. 105-128.

¥Généralisation relativiste des équations de Dirac, Académie Royale de Belgique, vol. 18 (1932), pp. 596-602.

$\S$ Diracsche und Schrödingersche Gleichungen, Berichte der Preussischen Akademie, 1933. 
part is a particular pseudo-cartesian lattice, but the application of the Lorentz transformation group to the whole system easily shows that by change of lattice the equations remain substantially unaltered. That is not the case in general relativity. Therefore, Dirac's equations must in my opinion be abandoned, without, it is understood, giving up the main progress realized by them in the domain of restricted relativity, which was to account for spin and magnetic perturbations.

14. Vector Equations of Schrödinger's Type with Additional Term. The equations I have recently proposed with the above aim can be briefly described as Schrödinger's vector equation (of the second order) with correcting term. It links together the 4 (covariant) components $\psi_{\mu}$ of a vector $\psi$, in the following way:

$$
S \psi_{\mu}+\chi_{\mu}{ }^{\nu} \psi_{\nu}=0 \quad(\mu=0,1,2,3),
$$

$S$ being Schrödinger's operation (8) and $\chi_{\mu}{ }^{\nu}$ a field tensor (function of the $x$ 's) defined by

$$
\chi_{\mu}{ }^{\nu}=C g_{\mu \rho} \epsilon^{\rho \nu \sigma \tau} F_{\sigma \tau},
$$

where $C$ is a constant, $F_{\sigma \tau}$ the (antisymmetric) electromagnetic tensor, and $\epsilon^{\rho v \sigma \tau}$ the contravariant $\epsilon$-tensor of Ricci, whose elements are 0 if the indices $\rho, \nu, \sigma, \tau$ are not all different, and, on this supposition, are equal to $1 /(-g)^{1 / 2}$, where $g=\left\|g_{\mu \nu}\right\|$, or to $-1 /(-g)^{1 / 2}$ according as the permutation $\rho \nu \sigma \tau$ is of even or odd order with respect to the fundamental permutation 0123 .

Equation (9) may be compared with the second-order consequences of Dirac's equations in pseudo-cartesian coordinates. Assuming

$$
C=e /\left(2 c h^{\prime}\right)
$$

we may verify that the two sets are identical for both typical cases of a magnetic or an electric field acting alone. For a general electromagnetic field, as well as for superposed effects of electromagnetism and gravitation, the two systems are no longer equivalent. $\dagger$

Herein lies, therefore, the possibility of discriminating, by some experiment, between the two as to which is to be preferred.

$\dagger$ Professor De Donder has kindly communicated to me his belief that, using his results, one may reach complete equivalence. 
Meanwhile it is to be observed that, under the general circumstances supposed here, the vector $\psi$ (whose components are ordinarily complex) is related to probability as follows: if $\psi_{\nu}^{*}$ denotes the complex conjugate of $\psi_{\nu}$, put

$$
P=g^{\mu \nu} \psi_{\mu} \psi_{\nu}{ }^{*},
$$

which is always a positive quantity, giving for a real vector $\psi$ its square length. We assume that $P d V_{4}$ is the probability for the electron being in an elementary volume (more precisely hypervolume)

$$
d V_{4}=(-g)^{1 / 2} d x^{0} d x^{1} d x^{2} d x^{3}
$$

of space-time manifold.

With the metric tensor $g_{\mu \nu}$, the elementary volume $d S$ of a surface $x^{0}=$ const. has the expression

$$
\left(-g g^{00}\right)^{1 / 2} d x^{1} d x^{2} d x^{3} .
$$

Our probability may therefore be written

$$
P d S d x^{0} /\left(g^{00}\right)^{1 / 2}
$$

or

$$
\operatorname{PcdS} /\left(g^{00}\right)^{1 / 2}
$$

for unit time ( $x^{0}$ meaning ordinary time multiplied by $c$ ).

15. Waves and Motion of Particles Mathematically Connected with a General System of Partial Differential Equations. According to Hugoniot and Hadamard, any normal system of partial differential equations, $\dagger$ for example (9), in the four functions $\psi_{0}, \psi_{1}, \psi_{2}, \psi_{3}$, determines uniquely, by a very simple rule, a first-order partial differential equation for an unknown function $z\left(x^{0}, x^{1}, x^{2}, x^{3}\right)$ attributing a more coarse wave appearance to the refined phenomenon actually governed by the normal system (9). For our system (9) the equation defining $z$ reduces to

$$
H=g^{\mu \nu} p_{\mu} p_{\nu} / 2=0, \quad\left(p_{\mu}=\partial z / \partial x^{\mu}\right),
$$

$\dagger$ A system is said to be normal if it involves as many equations as unknown functions, and is solvable with respect to certain highest derivatives. See my little volume (translated by G. Lampariello) Caratteristiche dei Sistemi Differenziali e Propagazione Ondosa (Bologna, Zanichelli, 1931), or the French translation (Paris, Alcan, 1932). 
or, solved with respect to $p_{0}$,

$$
p_{0}=-H\left(p_{1}, p_{2}, p_{3} \mid x^{0}, x^{1}, x^{2}, x^{3}\right),
$$

about which, without writing $H$ in full, it will be sufficient to say that $H$ is necessarily homogeneous of the first degree with respect to $p_{1}, p_{2}, p_{3}$. Its solution $z$, or better the surfaces $\sigma$ (in general variable with the time $x^{0}$ ) defined by

$$
z\left(x^{0}, x^{1}, x^{2}, x^{3}\right)=\text { const. }
$$

are possible wave fronts, in the sense that any of the $\sigma$ (and no other surfaces) may separate two regions in which different $\psi$ 's are solutions of (9), the two sets coinciding on the boundary.

If, as in the present case, the differential system verified by the $\psi$ 's is of the second order, then not only the functions themselves, but also their first derivatives, must be continuous across the $\sigma$, discontinuities affecting on the other hand second and higher derivatives.

Considerations which, at least in the ordinary pseudo-euclidean case, are well known, show that the equation $H=0$ has a very simple geometrical and physical meaning. It expresses the fact that a wave front, regarded as a moving surface in the surrounding three-dimensional space, advances (every element $d \sigma$ normally to itself) with the velocity of light. This displacement of any one of these elements $d \sigma$ gives rise to very expressive, though purely fictitious, point motions. It results from the general theory of characteristics that the point motions are regulated by the canonical system

$$
d p_{\mu} / d \tau=-\partial H / \partial x^{\mu}, d x^{\mu} / d \tau=\partial H / \partial p_{\mu}, \quad(\mu=0,1,2,3),
$$

with any independent variable $\tau$ (and the numerical specification $H=0)$; or in particular $\left(\tau=x^{0}\right)$, by

$$
d p_{\mu} / d x^{0}=\partial H / \partial x^{\mu}, d x^{\mu} / d x^{0}=-\partial H / \partial p_{\mu}, \quad(\mu=1,2,3),
$$

whose trajectories, as Cauchy's characteristics of (12), are the so called bicharacteristics of (9).

As you see, we have been able to associate in a very simple manner with (9)-as would be the same for more complicated physical processes - an undulatory face (waves of discontinuity) as well as a corpuscular one. This duality was first inferred by de Broglie with very striking intuition. It may be regarded, 
thanks to the mathematical theory just quoted, as a very general law of natural philosophy.

16. Specification of the Dualism, Waves-Particles, for Schrödinger's Equations. De Broglie's Wave-Length. Conclusion. The extensive statement of the preceding section is, however, rather abstract and agnostic. It affirms, for every physical theory, the admission of waves of discontinuity, mathematically resolvable into motion of discrete particles. But it gives no criterion for concrete coupling of the two single appearances. Attempts in this direction have been made by de Broglie himself, Schrödinger (\$10), Fermi and Persico, Maggi, and other scientists, leading to the conviction that no one-to-one correspondence between a wave (or a wave-packet) and a particle may be established without violating (as pointed out in $\$ 10$ ) the principle of indeterminacy.

We may, however, enlarge upon the question, and (as in $\$ 7$ for a rough treatment of electrons as electric current) attempt correspondences, not between individuals, but between sets of waves on the one hand and corpuscles on the other hand. To do this we must bear in mind some fundamental remarks concerning electrons or, analytically, the system (9):

(a) If it is not a single wave, then a wave train of discontinuity, passing across a little region $\Delta S$ in a little time $\Delta t$, as defined by (14), may be regarded as roughly equivalent to a set of electrons, every one of which may possibly be captured within $\Delta S$ and $\Delta t$.

(b) We shall admit, as in $\$ 7$, that such a set of electrons is indistinguishable from a continuous flow, and that this flow is connected by (14) to the ordinary Hamiltonian scheme of analytical mechanics, i.e., equivalent to the motion of a great number of discrete particles according to the canonical system $(15)$, or $\left(15^{\prime}\right)$.

This is not in contradiction with the analogous, but more elaborate, rule due to Schrödinger, in $\$ 11$. We started then from the Hamiltonian (5) suited for ordinary particles of mass $m$ and charge $e$. The actual Hamiltonian is $\mathfrak{S}$ of $(15)$, or more symmetrically, in space-time manifold,

$$
H \equiv g^{\mu \nu} p_{\mu} p_{\nu} / 2
$$


with the (integral) specification $H=0$ (see $\S 15$ and Appendix III). Now $H$ is nothing but a limiting case of (5) for particles very minute with respect both to mass and charge. In fact, drop in (5) all terms containing a factor $m$ or $e$ and you have (12).

By such assumptions we have attributed a statistical significance to the general dualism of waves and particles, analytically expressed by the mere fact that every normal system (especially Schrödinger's) possesses both characteristic surfaces and bicharacteristic lines.

(c) To obtain in our case concrete physical results, as de Broglie did, we still need yet one more postulate; and that is the attribution to the hypothetical particles, correlated with waves and therefore controlled by (15), of the exact mass $m$ belonging to each electron. $\dagger$

This enables us (see Appendix III) to get the very remarkable formula

$$
\lambda=h /\left(\left(g^{00}\right)^{1 / 2} m v\right),
$$

where $\lambda$ is the wave length of undulatory phenomena eventually accompanying electronic rays, $m$ the mass of any of the electrons forming the ray, $v$ the (mean) velocity of the flow, $h$ Planck's constant, and $g^{00}$, as in $\$ 14$, the pure time-like component of the contravariant metric tensor.

In restricted relativity, with reference to pseudo-cartesian coordinates, $g^{00}=1$, and (16) is precisely the relation discovered by de Broglie, before its experimental proof by Davisson and Germer, G. P. Thomson, and Rupp.

These hints may suffice, I hope, to justify my conviction that the analytical theory of characteristics of partial differential systems furnishes not only a sure and general frame work for the mathematical expression of the double, undulatory and corpuscular, appearance of any physical event, first foreseen and claimed by de Broglie; but even a statistical (if not as at first sought for, individual) connection between the two orders of things.

$\dagger$ This statement is not a consequence of (b), because according to the principle of indeterminacy, the continuous flow admitted there may not be regarded, from the physical standpoint, as made up of electrons. In fact by (b) we only introduced flow; the resolution of it into discrete particles, each of a given mass $m$, is a very natural, but independent hypothesis (postulate, in logical scheme). 


\section{APPENDIX I}

Symmetrical Treatment of the Einstein-DeDonder Principle of Variation. The principle of variation governing the motion of a particle of mass $m$ and charge $e$ in a general (gravitational and electro-magnetic) field, is

$$
\delta \int\left\{m c d s+\left(e f_{d} / c\right)\right\}=0,
$$

where, in general coordinates,

$$
d s^{2}=g_{\mu \nu} d x^{\mu} d x^{\nu}
$$

and $f_{d}$ means the Pfaffian

$$
f_{d}=\phi_{\mu} d x^{\mu},
$$

whose coefficients are the covariant components of the electromagnetic potential.

The function to be varied in (I.1) may obviously be multiplied by an arbitrary constant, without modification of the content of variational condition. The form adopted is a result of the desire to assure the exact dimensions of an action. Independently from this specification,

$$
m c d s+\left(e f_{d} / c\right)
$$

is homogeneous of the first degree with respect to the differentials $d x$; hence we may introduce an independent variable under the sign at will; e.g., the time-like $x^{0}$, or, more symmetrically, an auxiliary $\tau$. Denoting by a dot derivatives with respect to $\tau$, and putting

$$
\mathfrak{x}=g_{\mu \nu} \dot{x}^{\mu} \dot{x}^{\nu} / 2, \quad f=\phi_{\mu} \dot{x}^{\mu},
$$

we may present the variational equation (I.1) under the form

$$
\delta \int\left\{m c(2 \mathfrak{x})^{1 / 2}+(e f / c)\right\} d \tau=0 .
$$

At any rate it is to be remembered $\dagger$ that, owing to the firstdegree homogeneity, the four Lagrangian equations arising from

† See, for instance, Levi-Civita and Amaldi, Lezioni di Meccanica Razionale, vol. II, second part (Bologna, Zanichelli, 1927), p. 524. 
(I.5) are not independent, and therefore not sufficient to determine the four $x$ 's as functions of $\tau$; a circumstance which could be foreseen, as this auxiliary variable $\tau$ had been arbitrarily introduced. Hence we may add to (I.5) another relation, intended to fix the parameter $\tau$.

Excluding for the present eventual extremals of zero-length, i.e. solutions of (I.1) along which $d s^{2}$ is identically $=0$, we shall find it convenient to take just $d \tau=d s$, or by (I.2) and (I.4),

$$
2 \mathfrak{x}=1 \text {. }
$$

On account of this assumption, if we put for brevity

$$
L=m c \mathfrak{x}+(e f / c),
$$

(I.5) may be replaced by

$$
\delta \int L d \tau=0
$$

which, together with (I.6), is entirely equivalent to (I.5), (I.6).

The advantage of this transformation is that $L$ is no longer homogeneous of the first degree in the $\dot{x}$ 's. The Lagrangian system corresponding to (I.8) becomes of normal character in the $\ddot{x}$ 's; and Hamilton's reduction to canonical form may be performed as usual, through the introduction of moments $p_{\mu}$, conjugate to the $\dot{x}^{\mu}$, by the formulas

$$
p_{\mu}=\partial L / \partial \dot{x}^{\mu},
$$

which, in virtue of (I.7), (I.4), takes the form

$$
p_{\mu}=m c g_{\mu \nu} \dot{x}^{\nu}+\left\{e \phi_{\mu} / c\right\}, \quad(\mu=0,1,2,3) .
$$

To get the Hamiltonian $H(x \mid p)$, we have only to put

$$
H=p_{\mu} \dot{x}^{\mu}-L,
$$

eliminating the $\dot{x}$ 's by means of (I.9).

The resolution of (I.9) gives

$$
\dot{x}^{\mu}=g^{\mu \rho}\left(p_{\rho}-e \phi_{\rho} / c\right) /(m c),
$$

where every $g^{\mu \rho}$ is reciprocal to $g_{\mu \rho}$, i.e., the $g^{\mu \rho}$ are complementary minors (with due sign) of the $g_{\mu \rho}$ in the determinant of $\left\|g_{\mu \rho}\right\|$, divided by the determinant itself. 
The expression (I.7) of $L$ is broken up into the term $m c x$, homogeneous of the second degree in the $\dot{x}$ 's, and the term $e f / c$, homogeneous of the first degree. Accordingly

$$
p_{\mu} \dot{x}^{\mu}=\left(\partial L / \partial \dot{x}^{\mu}\right) \dot{x}^{\mu}
$$

reduces, by Euler's theorem, to

$$
2 m c \mathfrak{x}+(e f / c)
$$

and the Hamiltonian (I.10) to

$$
H=m c \mathfrak{x}
$$

where it remains only to replace the $\dot{x}$ 's by (I.11). From the original expression (I.4) of $\mathfrak{x}$, we get finally

(I.12) $H=m c \mathfrak{x}$

$$
\begin{aligned}
& =\left[\left(m c g_{\mu \nu}\right) \cdot g^{\mu \rho}\left\{p_{\rho}-\left(e \phi_{\rho} / c\right)\right\} / m c \cdot g^{\nu \sigma}\left\{p_{\sigma}-\left(e \phi_{\sigma} / c\right)\right\} /(m c)\right] / 2 \\
& =g^{\mu \nu}\left\{p_{\mu}-\left(e \phi_{\mu} / c\right)\right\}\left\{p_{\nu}-\left(e \phi_{\nu} / c\right)\right\} / 2(m c),
\end{aligned}
$$

which is the announced form.

The additional condition (I.6) gives

$$
H=m c / 2 \text {, }
$$

which must be associated to the canonical system

$$
\dot{p}_{\mu}=-\partial H / \partial x^{\mu}, \quad x^{\mu}=\partial H / \partial p_{\mu}, \quad(\mu=0,1,2,3),
$$

in order to get a differential system completely equivalent to (I.6), (I.8), or to the original variational principle (I.5)together with (I.6).

As $H$ does not involve explicitly the independent variable $\tau$, the differential system (I.14) admits the classical integral

$$
H=\text { const. }
$$

We see therefore that the canonical form just arrived at has the advantage, in addition to others, that the additional equation (I.13) requires only a particularization of the constant value of $H$.

Extremals of Zero-Length. In the particular case, previously excluded, $d s^{2}=0$, the equation

$$
2 \mathfrak{x}=0
$$


is by hypothesis satisfied along the corresponding class of solutions, and then we may no longer particularize the independent variable $\tau$ by a contradictory equation as $2 \mathfrak{x}=1$. But conversely, leaving $\tau$ undetermined, we may proceed exactly as before, the only difference being that the constant 1 of (I.6) is here replaced by 0 . The conclusion is that the canonical system (I.14) is still valid, the constant value of $H$ being 0 instead of $m c / 2$.

\section{APPENDIX II}

Illustrative Examples of Several Devices by Which Transformations in General Coordinates May be Performed. Let us consider, with reference to two independent variables $x$ and $y$, the differential equations

$$
\partial \phi / \partial x-\partial \psi / \partial y=0, \quad \partial \phi / \partial y+\partial \psi / \partial x=0,
$$

which express conditions that $\phi+i \psi$ be a (monogenic) function of the complex variable $x+i y$.

How are these equations to be transformed, in passing to new variables $x^{1}, x^{2}$, bound to $x, y$ by given relations?

If no additional condition is added, the question admits, as we shall see, of several solutions, all substantially equivalent, but leading to different forms of the equations referred to new variables.

In order to obtain a clear understanding in this regard, let us adopt successively three criteria, all reasonable, for the transformation of (II).

(a) As an entirely suitable convention, we may assume that the functions $\phi$ and $\psi$ behave both as invariants. This means that, when the $x, y$ are replaced by the new variables $x^{1}, x^{2}$, the transformed $\phi, \psi$ are the same as before, with the understanding, however, that the $x, y$ must be replaced by their expressions as functions of $x^{1}, x^{2}$.

Let us confine ourselves at first to isometric transformations, i.e. those arising from a unique relation $z^{\prime}=f(z)$, where $z=x+i y$ as before, $z^{\prime}=x^{1}+i x^{2}$, and $f$ is an analytic function. Then the transformed $\phi\left(x^{1}, x^{2}\right), i \psi\left(x^{1}, x^{2}\right)$ are still the real and imaginary parts of a function of the complex variable $z^{\prime}$, and the equations (II) become 


$$
\partial \phi / \partial x^{1}-\partial \psi / \partial x^{2}=0, \quad \partial \phi / \partial x^{2}+\partial \psi / \partial x^{1}=0,
$$

thus preserving the form (II).

(b) We pass now to general changes of variables (no longer isometric), still assuming for $\phi$ and $\psi$ the law of invariance.

To get the equations corresponding to (II) in the new variables $x^{1}, x^{2}$, we have only to introduce, instead of $\partial / \partial x, \partial / \partial y$, the operators

$$
\begin{aligned}
& \left(\partial x^{1} / \partial x\right)\left(\partial / \partial x^{1}\right)+\left(\partial x^{2} / \partial x\right)\left(\partial / \partial x^{2}\right), \\
& \left(\partial x^{1} / \partial y\right)\left(\partial / \partial x^{1}\right)+\left(\partial x^{2} / \partial y\right)\left(\partial / \partial x^{2}\right) .
\end{aligned}
$$

The transformed equations then look rather complicated, in no way resembling the original equations. But it is possible to give them an invariant structure by the aid of geometric subsidiary elements. If we think of $x, y$ as being cartesian coordinates, and introduce the square length

$$
d s^{2}=d x^{2}+d y^{2},
$$

we shall have, in general coordinates $x^{1}, x^{2}$,

$$
d s^{2}=a_{\mu \nu} d x^{\mu} d x^{\nu}
$$

the coefficients $a_{\mu \nu}(\mu, \nu=1,2)$ thus providing the metric tensor.

The cartesian lattice $x=$ constant, $y=$ constant is formed by two congruences of (straight) lines, each of which has a proper geometrical meaning. The partial derivatives $\partial / \partial x, \partial / \partial y$ may be regarded as directional derivatives $d / d s_{1}, d / d s_{2}$ along the lines $y=$ constant, and $x=$ constant, respectively. They obtain in this way an invariant determination which may be easily put in explicit form with respect to our generic (curvilinear) coordinates $x^{1}, x^{2}$. We need only introduce, with reference to these coordinates, the parameters $\lambda_{1}{ }^{\mu}, \lambda_{2}{ }^{\mu}(\mu=1,2)$ of the lines $y=$ constant, $x=$ constant, respectively. We have then

$$
\partial / \partial x=d / d s_{1}=\lambda_{1}{ }^{\mu} \partial / \partial x^{\mu}, \quad \partial / \partial y=d / d s_{2}=\lambda_{2}{ }^{\mu} \partial / \partial x^{\mu},
$$

and (II.1) takes the form

$$
d \phi / d s_{1}-d \psi / d s_{2}=0, \quad d \phi / d s_{2}+d \psi / d s_{1}=0,
$$

which is, in the explained sense, completely invariant.

In the particular case of an isometric transformation, these equations may be linearly combined so as to assume the previous form (II.1). 
(c) Instead of regarding $\phi$ and $\psi$ as invariant functions, it is possible to make other assumptions; we may for instance suppose that, when variables are changed, not the single $\phi$ and $\psi$, but the differential form

$$
\phi d x-\psi d y
$$

has invariant character. This means that, if in general coordinates $x^{1}, x^{2}$ the linear form $\phi d x-\psi d y$ becomes

$$
v_{1} d x^{1}+v_{2} d x^{2}
$$

the coefficients $v_{1}, v_{2}$ are to be considered as the transforms of $\phi$ and $\psi$ (law of covariance). The explicit formulas are

$$
\begin{aligned}
& v_{1}=\phi \partial x / \partial x^{1}-\psi \partial y / \partial x^{1} \\
& v_{2}=\phi \partial x / \partial x^{2}-\psi \partial y / \partial x^{2} .
\end{aligned}
$$

On this assumption $v_{1}, v_{2}$ may be interpreted as covariant components of a vector $v$, precisely of that vector which had, in the original cartesian coordinates, the components $\phi$ and $-\psi$.

Let us now proceed to the transformation of the differential system (II) by introduction into it of new variables $\left(x^{1}, x^{2}\right.$ instead of $x, y)$ and also new functions $\left(v_{1}, v_{2}\right.$ instead of $\left.\phi,-\psi\right)$.

The second equation of (II) is simply the condition for the Pfaffian

$$
\phi d x-\psi d y=v_{1} d x^{1}+v_{2} d x^{2}
$$

to be an exact differential. As this property always belongs to the same Pfaffian, whatever variables are adopted, we must have

$$
\partial v_{1} / \partial x^{2}-\partial v_{2} / \partial x^{1}=0,
$$

which supplies one of the transformed equations. In vectorial language it expresses the fact that the vector-field $v$ is a conservative one, or that a function $V$ of position exists, whose gradient is $\boldsymbol{v}$.

To complete without direct calculation the transformation of (II), it will be useful, here again, to appeal to the euclidean metric, defined by

$$
d s^{2}=d x^{2}+d y^{2}=a_{\mu \nu} d x^{\mu} d x^{\nu} .
$$


On account of this metric we may introduce, at any point $x, y$, besides $v$, a second vector $w$ through a rotation of $90^{\circ}$ (in the positive sense of the cartesian lattice, $O x y)$. The cartesian components of $w$ are then $\psi, \phi$; and the first of the equations (II) is only the expression of the fact that the vector-field $w$ admits a potential function. It may therefore be transformed, as was the other, in general coordinates $x^{1}, x^{2}$, giving

$$
\partial w_{1} / \partial x^{2}-\partial w_{2} / \partial x^{1}=0,
$$

where $w_{1}, w_{2}$ denote clearly the covariant components of $w$ to be calculated from (II.3) (by writing there $\psi$ and $\phi$ instead of $\phi$ and $-\psi)$.

Synthetically we may conclude that, by the introduction of euclidean metric and, at every point, of a second vector $w$ (orthogonal to $v$ and of equal length), both equations (II) have invariant interpretation.

They are merely the conditions that the fields $v$ and $w$ each admit a potential function, that is to say are irrotational, or finally that they have vanishing curl round any circuit (reducible to a point, within the field).

(d) But we may go a step further and reach another invariant interpretation, which requires only one of the two vector-fields $\boldsymbol{v}$ and $\boldsymbol{w}$ : say $\boldsymbol{v}$. In order to see this we need only to remember that $w$ is perpendicular to $v$ and has equal length. Hence, along a generic curve $s$, if $d s$ and $d n$ denote the elements of arc and of the normal (oriented as $d x, d y$ ), we have for the components of $v, w$,

$$
v_{s}=w_{n}, \quad v_{n}=-w_{s},
$$

and therefore, for any arc $s$,

$$
\int_{s} v_{s} d s=\int_{s} w_{n} d s, \quad \int_{s} v_{n} d s=-\int_{s} w_{n} d s,
$$

so that the vanishing of the curl of one vector, around any closed line, implies the vanishing of the flow of the other vector, and conversely. We may consequently replace the statement under (c), equivalent to (II), by the following:

In the vector-field $v$, curl and flow are both zero (i.e., the field is at the same time conservative and solenoidal). 
In the usual vector symbolism we may write

$$
\operatorname{rot} v=0, \quad \operatorname{div} v=0,
$$

or explicitly

$$
v_{12}-v_{21}=0, \quad a^{\mu \nu} v_{\mu \nu}=0,
$$

where $v_{\mu \nu}$ are covariant derivatives of $v_{\mu}$ with respect to the euclidean form (II.2) and $a^{\mu \nu}$ coefficients of its reciprocal. The first of these equations is not only equivalent to, but even identical with (II.5), by a known relation between covariant and ordinary derivatives.

Apart from (II.1), which concern only isometric transformations, the other forms (II.3); (II.5); (II.6); (II.7); or (II.8), in which the original system (II) may be represented referred to general coordinates, are all substantially equivalent, in the sense that the two equations belonging to one of them are linear combinations of two others, with at most further linear substitution in the two dependent variables.

In the abstract the various systems arrived at are on the same footing. Only particular reasons, arising from the nature of things represented by the initial equations (II), or ease of calculation, may lead to the preference of one form to another.

Remark: To get from (II) any one of the transformed systems (II.3); (II.5); (II.6); (II.7); (II.8), in general coordinates, we have treated the original variables $x, y$ as cartesian coordinates, employing the auxiliary $d s^{2}=d x^{2}+d y^{2}$. It is to be noted that every transformed system has a perfectly defined meaning even if the $d s^{2}=a_{\mu \nu} d x^{\mu} d x^{\nu}$ involved is no longer euclidean. Then there do not exist special coordinates $x, y$ for which the $d s^{2}$ takes the typical form $d x^{2}+d y^{2}$, and therefore the general forms just quoted are no longer transforms of (II), though equivalent to each other. This happens often in the physical applications, where differential systems (like (II) but much more complex) are first established from premises connected with euclidean space, and then inferred for spaces of any metrical structure, through their transforms in general coordinates. Here we have Ricci's device for passing from euclidean to any metric. It rests on the belief that Riemann's curvature does not directly affect the natural laws; thus the passage to any metric becomes uniquely determined (at least for the more common theories). 


\section{APPENDIX III}

Atomic Wave-Trains. Analysis and Physical Generalization of de Broglie's Inferences. Let us consider a space-time manifold $V_{4}$.

If $\tau$ represents any auxiliary independent variable, parametric equations

$$
x^{\mu}=x^{\mu}(\tau), \quad(\mu=0,1,2,3),
$$

define a curve in $V_{4}$, or a motion of a point $P$. If, along this motion, $d s^{2}>0$, as for material bodies, it is convenient and customary to take at once $d \tau=d s$, the motion being thus referred to the proper time of $P$. But if $d s^{2}=0$, which means that the four-dimensional path is of zero-length (see end of Appendix I), it is meaningless to appeal to $d s$, and the introduction of some other parameter $\tau$ becomes unavoidable. At any rate the differential quotients of the $x^{\mu}$ 's with respect to $\tau$ (which, for a proper motion, that is, for $d s^{2}>0$, are proportional to the $d x^{\mu} / d s$ ) may be regarded as the contravariant components of a fourvector $w$.

Let us put

$$
L(x \mid \dot{x})=g_{\mu \nu} \dot{x}^{\mu} \dot{x}^{\nu} / 2, \quad(\operatorname{dot} \text { meaning } d / d \tau),
$$

and

$$
p_{\mu}=\partial L / \partial \dot{x}^{\mu}=g_{\mu \nu} \dot{x}^{\nu}, \quad(\mu=0,1,2,3),
$$

obtaining in this way the covariant components of the same vector $w$.

Obviously we may regard $w$ as the sum of two vectors having respectively $\left(p_{0}, 0,0,0\right)$ and $\left(0, p_{1}, p_{2}, p_{3}\right)$ as covariant components: $w$ is then resolved into two vectors $w_{0}$ and $w_{1}$, the former perpendicular and the latter tangent to the spacial section $x^{0}=$ constant, through $P . \dagger$

We know from the restricted relativity, where $x^{0}=c t$ and the $p$ 's are identical with the corresponding $\ddot{x}$ 's, that the ratio of the absolute values of $w_{0}$ and $w_{1}$,

$\dagger$ It would be equally permissible to decompose $w$, through its contravariant components, into the two vectors $\left(\dot{x}^{0}, 0,0,0\right),\left(0, \dot{x}^{1}, \dot{x}^{2}, \dot{x}^{3}\right)$. Then the former has the direction of the time-like line $x^{0}\left(x^{1}=\right.$ constant, $x^{2}=$ constant, $x^{3}=$ constant), the latter still being tangent to the spacial section. The two (covariant and contravariant) decompositions coincide if, and only if, the $x^{0}$-lines are orthogonal to the surfaces $x^{0}=$ constant, i.e., if $g_{01}=g_{02}=g_{03}=0$ : 


$$
w_{0}=\left|p_{0}\right|, \quad w_{1}=\left|\left(p_{1}^{2}+p_{2}^{2}+p_{3}^{2}\right)^{1 / 2}\right|,
$$

behaves as energy divided by $c$ to momentum $q$ of the moving point, i.e.,

$$
w_{0} / w_{1}=E /(c q) .
$$

For a general $V_{4}$ we are naturally led to assume that (III.3) still holds as a mechanical interpretation of the geometrical decomposition of $w$. On account of the fourfold metric

$$
d s^{2}=g_{\mu \nu} d x^{\mu} d x^{\nu},
$$

$g^{\mu \nu}$ being the reciprocal elements, we have for the merely temporal vector $w_{0}$, of covariant components $\left(p_{0}, 0,0,0\right)$, the square length

$$
w_{0}=g^{00} p_{0}^{2} .
$$

For the merely spacial vector $w_{1}$ of covariant components $\left(0, p_{1}, p_{2}, p_{3}\right)$, we must appeal to the metric, subordinated by the former $d s^{2}$ in a section $x^{0}=$ constant, i.e., to

$$
-d l^{2}=\sum_{1}^{3}{ }_{\mu \nu} g_{\mu \nu} d x^{\mu} d x^{\nu}
$$

It will accordingly be convenient to introduce, besides the $g^{\mu \nu}$, the coefficients $g^{\prime \mu \nu}$ of the ternary form reciprocal to $d l^{2}$ (which coincide with $-g^{\mu \nu}$ only when $g_{01}=g_{02}=g_{03}=0$ ). The three-dimensional contravariant components $p_{\mu}{ }^{\prime}$ of $w_{1}$ are then

$$
p^{\prime \mu}=\sum_{1}^{3}{ }_{\nu}{ }^{\mu \nu} p_{\nu}
$$

and therefore

$$
w_{1}^{2}=\sum_{1}^{3}{ }_{\mu} p_{\mu} p^{\prime \mu}=\sum_{1}^{3}{ }_{\mu \nu} g^{\prime \mu \nu} p_{\mu} p_{\nu}
$$

Now let us especially suppose that the $p_{\mu}$ verify equation (12) of the text, i.e.,

$$
H(p \mid x)=g^{\mu \nu} p_{\mu} p_{\nu} / 2=0,
$$

which, owing to the identity

$$
2 L=g_{\mu \nu} \dot{x}^{\mu} \dot{x}^{\nu}=g^{\mu \nu} p_{\mu} p_{\nu}=2 H,
$$


expresses the fact that the four-vector $w$ is of zero length. Furthermore, if we treat the $p_{\mu}$ as derivatives of an unknown function $z\left(x^{0}, x^{1}, x^{2}, x^{3}\right)$, equation (III.8) may be solved with respect to $p_{0}$ and we get equation (13) of the text, that is,

$$
p_{0}+\mathfrak{S}\left(p_{1}, p_{2}, p_{3} \mid x\right)=0 .
$$

Let us fix our attention on any particular solution

$$
z\left(x^{0}, x^{1}, x^{2}, x^{3}\right)
$$

of (III.8). By virtue of the homogeneity, in $z$, any function $f(z)$ is also a solution.

In the neighborhood of any point $P$ of $V_{4}$, that is, of any system of values $x^{\mu}$, we have

$$
d z=p_{0} d x^{0}+\sum_{1}^{3} p_{\mu} d x^{\mu}
$$

$d x^{0}, d x^{\mu}$ being arbitrary. Let us consider $x^{1}, x^{2}, x^{3}$ separately from $x^{0}$, and look provisionally at $z$ as depending on $x^{1}, x^{2}, x^{3}$, while $x^{0}$ acts as parameter. Its derivatives $p_{\mu}(\mu=1,2,3)$ are the covariant components of the vector $w_{1}$. We have therefore on one hand

$$
w_{1}=\operatorname{grad}^{\prime} z,
$$

the accent alluding to a mere three-dimensional gradient; and besides, from (III.7),

$$
\Delta^{\prime} z=\sum_{1}^{3}{ }_{\mu \nu} g^{\mu \nu} p_{\mu} p_{\nu}=w_{1}^{2},
$$

$\Delta^{\prime} z$ being Beltrami's parameter of the function $z$ with respect to the spacial metric having $d l^{2}$ as square element of line.

Let us now resume using from (III.6) the contravariant components $p^{\prime \mu}$ of $w_{1}$ (in this metric). The parameters of the corresponding direction are

$$
p^{\mu} / w_{1}=p^{\prime \mu} /\left(\Delta^{\prime} z\right)^{1 / 2} .
$$

The spacial displacement $d x^{\mu}$ in (III.10) may be resolved into two components: the one of algebraic value $-d n$, directed along $\boldsymbol{w}_{1}=\operatorname{grad}^{\prime} z$, and therefore of contravariant components

$$
-d n\left(p^{\prime \mu} / w_{1}\right)
$$


and the other perpendicular to $w_{1}$. This latter contributes nothing to the scalar product by $w_{1}$, i.e., to the sum $\sum_{\mu=1}^{3} p_{\mu} d x^{\mu}$. Therefore we may write, by virtue of (III.7), (III.4),

$$
\begin{aligned}
\sum_{1}^{3} p_{\mu} d x^{\mu} & =-w_{1} d n, \\
p_{0} d x^{0} & =\left(w_{0} /\left(g^{00}\right)^{1 / 2}\right) d x^{0},
\end{aligned}
$$

so that, by (III.10), in the infinitesimal neighborhood of $P$, the expression of our integral $z$ reduces to

$$
z=z_{p}+\left(w_{0} /\left(g^{00}\right)^{1 / 2}\right) d x^{0}-w_{1} d n .
$$

Hence we recognize that, in the immediate neighborhood of any instant $x^{0}$ and place $x^{1}, x^{2}, x^{3}$ (our point $P$ of $V_{4}$ ), z may be regarded as a linear function of only two independent arguments: the one, $d x^{0}$, or $d x^{0} / c$, expressing (this latter ordinary) time, and the other, $d n$, expressing length in the spacial direction of $\operatorname{grad}^{\prime} z$.

In the same infinitesimal range, with

$$
z_{p}+\left(c w_{0} /\left(g^{00}\right)^{1 / 2}\right) d x^{0} / c-w_{1} d n,
$$

any function whatever of this argument is again, as observed just above, a solution of the same homogeneous equation (III.8) or (III.9).

We may assume in particular as a solution

$$
z=a e^{2 \pi i \alpha\left\{\left(c w_{0} /\left(g^{00}\right)^{1 / 2}\right) d x^{0} / c-w_{1} d n\right\}},
$$

where

$$
a=e^{2 \pi i z_{P}}
$$

and $\alpha$ is an arbitrary constant (absolute, or depending upon $P$ ). This wave-function $z$ in this form is familiar as the ordinary representation of vibratory progressive waves; therefore

$$
\alpha c w_{0} /\left(g^{00}\right)^{1 / 2}
$$

is to be interpreted as instantaneous frequency $\nu$, and $1 /\left(\alpha w_{1}\right)$ as local wave-length $\lambda$. At any rate the velocity of propagation $V$ has the value

$$
V=\lambda \nu=\left(c w_{0}\right) /\left(\left(g^{00}\right)^{1 / 2} w_{1}\right)
$$

independent of the arbitrary constant $\alpha$. 
Now one can associate with wave-trains of this kind the corresponding bicharacteristics (15). The $p_{\mu}(\mu=0,1,2,3)$ just dealt with are then to be interpreted as covariant components of a fourfold vector $w$, related by (a), (b), (c) of $\S 16$ to the motion of particles of mass $m$. We have accordingly from (III.3) the corpuscular interpretation of $w_{0} / w_{1}, E /(m c v)$, where $v$ and $E$ represent their (mean) individual velocity and energy.

Introducing this value of $w_{0} / w_{1}$ in (III.13), we have

$$
\lambda \nu=E /\left(\left(g^{00}\right)^{1 / 2} m v\right),
$$

and finally, if $\nu$ and $E$ are correlated by Planck's law $E=h \nu$,

$$
\lambda=h /\left(\left(g^{00}\right)^{1 / 2} m v\right),
$$

which is the expression of de Broglie's wave-length, extended from pseudo-euclidean to any space-time manifold, with explicit statement of the physical assumptions on which it rests.

The University of Rome 